\title{
Evaluating the Behavioral and Developmental Interventions for Autism Spectrum Disorder Karthik.K ${ }^{\mathrm{a}}$,Akila.R ${ }^{\mathrm{b}}$, Sophiga.Mc ${ }^{\mathrm{c}}$, Priyadharshini $\mathbf{R}^{\mathrm{d}}$, Karthiga $\mathbf{N}^{\mathrm{e}}$
}

aDepartment of Computer Science and Engineering ,M.Kumarasamy College of Engineering, Karur, Tamil Nadu, India -639113 b,c,d,e Department of Computer Science and Engineering, M.Kumarasamy College of Engineering, Karur, Tamil Nadu, India -639113 karthikk.cse@mkce.ac.in,selviakila29@gmail.com, sophiga1999@gmail.com,priyacse000@gmail.com, karthickacse55@gmail.com

Article History: Received: 11 January 2021; Accepted: 27 February 2021; Published online: 5 April 2021

\begin{abstract}
It is currently impossible to estimate the number of users who are experiencing Mental Depression symptoms. Today's recognition is highly reliant on observation of users' behaviours. This paper suggests a framework for considering behaviour factor relationships and categorising them using association-based classification (CBA).Patient profiles from two Thai emergency clinics were utilized in our trials. Experts requested this data into two groupings: Autism and Pervasive Developmental Disorder - Not Otherwise Specified (PDD-NOS). Our discoveries uncover an assortment of charming conduct patterns in individuals with mental imbalance.These findings provide doctors with invaluable knowledge for future research into early autism symptom intervention. Our project aims to develop a data processing method that will aid doctors in diagnosing patients later on.
\end{abstract}

Index Terms: Watchwords Data Mining; Mental Depression; Analysis of clinical information; order dependent on affiliations.

\section{Introduction}

Mental sorrow is a cerebrum problem that makes clients' initial years be broken and postponed. Mental discouragement is a typical conduct grouping plan zeroed in on debilitations in three areas: social connection, correspondence, and a different scope of wants and practices [1]. The irregularity ordinarily introduces itself before the client arrives at the age of three, making location troublesome. Pretty much $10 \%$ of people with chemical imbalance have a particular reason, which is most usually delicate X condition, tuberous sclerosis, and chromosomal irregularities, while the remainder of individuals with idiopathic Psychiatric DEPRESSION don't. In reality, analysis is made by looking at a patient's conduct utilizing a couple of basic conventions. The most by and large used investigation governs today are the Diagnostic and Statistical Manual of Mental Disorders, Fourth Edition (DSM-IV) [1] and the Autism Diagnostic Observation Schedule (ADOS) [3]. It's also essential to see between the two. (Mental imbalance and Pervasive Developmental Disorder) Not in any case expressed (PDD-NOS). Manifestations of PDD-NOS are like those of mental imbalance, in spite of the fact that they are regularly less serious.

A few patterns in medically introverted babies' conduct, like an absence of socialization, obliviousness of one's own name, and an absence of looking and grinning at others, might be utilized as a beginning stage for more investigation. As indicated by a report distributed in [4], around $66 \%$ of 67 mentally unbalanced clients experience outrageous fits, and around $33 \%$ of the individuals who have had fits of rage in the past have been forceful. A postponement in normal discourse improvement and low achievement in exercises including complex language use, like cognizance and surmising, are two other important social attributes. [5].

Since previous updates, no particular activity appears to point directly to MENTAL DEPRESSION, but there are some recurring behavioural trends that merit further study. As a result, with a large enough sample of patient behaviour data, it could be possible to establish a connection between certain behaviours and autistic symptoms. This paper explores data mining methods, which are meant to provide doctors with a set of tools to help them analyse patient data intelligently.

We tried to derive forms from behavioural data and establish a classifier for patient behaviours in this research. An associative classification system is the method suggested.A grouping based affiliation (CBA) approach is utilized to arrange conduct patterns of mental imbalance and PDD-NOS clients. Our findings provide valuable information that can be used by controller practitioners in the future to choose suitable interventions, which may assist autistic people in working more effectively in society and allow for early diagnosis and intervention.

In this study, 140 patients from two Thai hospitals were used as test subjectsAutism or PDD-NOS has been diagnosed with many of the patients

Coming up next is the format of the paper: Section II remembers an audit of past examinations for clinical information mining just as a foundation report on affiliated grouping. The issue and related wordings are depicted in Section III. In Section IV, the examination approach is tended to. In Section V, the exploratory outcomes and conversations are examined. The hypothesis and rules for future assessment are found in Section VI.

\section{Presentation and Associated Researches}

\subsection{Information Mining for Medical Data Analysis}

Several researchers have attempted to analyse medical data using various datain recent years, mining methods. The results of behaviour therapy for autism disorder were studied in [6]. To find the various phases of therapy, decision trees (ID3) and association rule mining were 
used. They used a ten percent help and a ninety percent assurance scale to evaluate the rules.These rules will predict the degree to which acceptable and unacceptable behaviours are present.Analysts in [7] expected 58 patients with fringe cellular breakdown in the lungs illness utilizing affiliation rule.Sex, age, form, and ground-glass density were all factors included in the diagnosis. The material was then used to build an expert community that used a network of diagnostic expertise to execute clinical tasks.

[8] directed one more examination on the point. A choice tree, Naive Bayes, and Neural Networks were utilized to make a calculation to arrange covered up examples and connections in medical services information. The utilization old enough, pulse, and glucose levels to decide the probability of a patient contracting coronary illness has all been fruitful. By utilizing order mining, information is planned into predefined gatherings. A conclusion characterization classifier was created utilizing this apparatus. The classifier is created utilizing AI and authentic clinical records ("preparing"). It could be utilized to order mysterious clinical information into condition bunches ("testing").Treatment that is viable ought to be finished.

Associative classification (AC) has been suggested as a method for classifying data. The method distinguished itself from traditional classification methods by emphasising attribute relationships. Functional AC to a drug screening demonstration, according to research in [9]. The findings were found to be superior to current classification strategies like C4.5.

\subsection{Associative Classification}

Effective classification techniques provide an associative classification (AC) relation in association rule mining. The chase for exacting principles that are connected to class marks as opposed to objects. A basic in the event that technique is utilized to portray the impacts of AC.On the premise of $\mathrm{AC}$, an assortment of strategies have been proposed. We took a gander at three regular calculations: order by affiliation (CBA) [9], arrangement by various guidelines (CMAR) [10], just as depiction by affiliation decides that foresee (CPAR) [11].Services given by the CBA During the affiliation cycle, theApriori calculation is utilized, while CMAR utilizes FP-development. Utilizing a different principle strategy, the CMAR and CPAR show up at outright laws. Numerous guidelines, notwithstanding, can make various cases, for example, coordinating with a few standards with various class marks. In CMAR and CPAR, this issue presently can't seem to be tended to.

[12] connected various distinctive AC calculations together. CPAR was discovered to be ideal for an enormous dataset on the grounds that it required some investment to create rules. Everything calculations can deliver effective classifiers with a proper blunder rate regarding precision.

We just utilized a couple of patient records in our investigation. Thus, execution time isn't an issue. The calculation will deliver extra principles by utilizing CBA.

\subsection{Association-based grouping}

Finding concludes that can reliably expect classes is the place of alliance based assembling (CBA). It contains two critical parts: (1) a standard generator (CBA-RG) for making a full plan of class association rules (CARs), and (2) a classifier producer (CBA-CB) for cutting the course of action of CARs and conveying a classifier.

In the underlying advance, CBA-RG uses the Apriori count to create all progressive rule things and find all principles with maintains more essential than the customer portrayed edge. A standard thing can be composed as X Y, with X being the predecessor (IF-part) and Y being the ensuing (THEN-part).Instead of a trait set as seen in Apriori, the THEN-part of a standard article is a class mark. From that point forward, prune rule objects are placed into CARs and arranged by trust esteems. The initial step is rehashed before the IF-part passes a specific boundary.

The subsequent stage incorporates perusing and positioning need rules from the entirety of the preparation cases in the CARs list. The most raised scoring rules are then used to gather a classifier. The most standard classes that haven't been picked as a default class.

An estimation of the base help edge altogether affected the precision classifier in the past examination. At the point when the limit is set at 1-2 percent, CBA's classifier would be more precise than C4.5's [13, 14].

\section{DEFINITION OF THE PROBLEM}

\subsection{Assessment of Actions}

The investigations utilize 32 essential boundaries to order the patients. Table I records the entirety of the signs found in the conduct test utilized in this article.

\begin{tabular}{|c|l|}
\hline Thing No. & Thing Description \\
\hline 1 & There is no conveyed in language or talk that is conceded. \\
\hline 2 & Language is seriously hampered \\
\hline 3 & A significant decrease in one's ability to hit up a discourse with outsiders. \\
\hline 4 & A perceptible lessening in one's capacity to speak with others. \\
\hline 5 & Utilization of jargon that is generalized and redundant \\
\hline
\end{tabular}




\begin{tabular}{|c|c|}
\hline 6 & Peculiar expressing \\
\hline 7 & Utilizing fundamental signals to impart in an unconstrained way is absent. \\
\hline 8 & Unseemly utilization of an assortment of facial motions for enthusiastic reactions \\
\hline 9 & Inability to show an interest in something by pointing with a finger \\
\hline 10 & At the point when a kid is in agony or harmed, they don't specify it to their folks. \\
\hline 11 & Acknowledgment of others' pleasure or trouble is missing or fractional. \\
\hline 12 & Inability to grin when others grin \\
\hline 13 & Inability to answer when drawn nearer by name \\
\hline 14 & At the point when his folks are playing with him, he neglects to visually connect. \\
\hline 15 & Inability to show guardians things to exhibit a point \\
\hline 16 & The individual in question is an individual who lives in their own little universe. \\
\hline 17 & Games that can be played alone are supported.. \\
\hline 18 & Guardians' endeavors to play with their youngsters are met with an improper reaction. \\
\hline 19 & Response unsuitable to different kids being or playing together \\
\hline 20 & Powerlessness to get different children to play a simple game \\
\hline 21 & Any clients' deficiency of confidence drives them to renege. \\
\hline 22 & Impassion of others \\
\hline 23 & Inability to connect with others in simple play \\
\hline 24 & Playing with various kinds of toys exhausts you. \\
\hline 25 & Playing with different sorts of toys debilitates you. \\
\hline 26 & Improperly playing with little toys, for example, mouthing, playing, or dropping them \\
\hline \multirow[t]{2}{*}{27} & \\
\hline & Examples with strangely solid or centered restricted interest. \\
\hline 28 & Distraction with object pieces \\
\hline 29 & $\begin{array}{l}\text { Emphasis on such practices or ceremonies, like wearing a particular coat or guaranteeing that the entirety of their toys } \\
\text { are in the legitimate area }\end{array}$ \\
\hline 30 & For significant stretches of time, twirling or spinning himself around \\
\hline 31 & For significant stretches of time, twirling or spinning himself around \\
\hline
\end{tabular}

\section{Table I: Behavior Test}

\subsection{Dataset}

140 medical reports make up the clinical results. Every record is based on a behavioural statement and an interview that meets the 32 requirements mentioned above. Specialists grouped every understanding's reports as mental imbalance or PDD-NOS. There are around 110 chemical imbalance cases and 30 PDD-NOS cases in the data set. The patients went in age from 17 to 145 months. There were 121 guys and 19 females in the school.

Every measurement's attribute can be either "current" (impairment behaviour is manifested) or "absent" (impairment behaviour is not manifested) (something else).

\subsection{Issue Statement}

We describe a lot of suggestions that will be used in the examination in our report. This section covers a request for mathematical decrees subject to the association methodology.

Definition 1: Let $\mathrm{D}=\mathrm{T} 1, \mathrm{~T} 2, \ldots \mathrm{Tn}$ address a patient exchange dataset, and $\mathrm{I}=\mathrm{I} 1, \mathrm{I} 2, \ldots, \mathrm{Im}$ address the ascribes in $\mathrm{D}$. The arrangement of class names is $\mathrm{Y}=\mathrm{Y} 1, \mathrm{Y} 2, \ldots, \mathrm{Yp}$. In any occasion one ascribes from set I and one class mark from Y make up each peaceful trade Ti.

Definition 2: Allow itemset to be an assortment of characteristic qualities that aren't no different either way. Let ruleitem R: X Yi be 
the standard for distinguishing designs in a conduct test, with $\mathrm{X}$ being an itemset $\mathrm{X} \mathrm{I}$ and Yi being a variable.

Definition 3: Let the quantity of exchanges containing itemset $X$ and having a place with class Yi be the assistance check (suppcount) of ruleitem R. Let the quantity of exchanges that contain itemset $\mathrm{X}$ be a genuine occasion (actoccr). Let conf(R) be the degree of trades in $\mathrm{D}$ that satisfy the standard antecedent and moreover have a Yi class mark.

Definition 4: A standard thing $\mathrm{R}$ can be known as an incessant principle thing if and just

in the event that $\operatorname{supp}(\mathrm{R})=(\operatorname{supp} \operatorname{count}(\mathrm{R}) /|\mathrm{D}|)_{-}$min supp, where $|\mathrm{D}|$ is the size of the dataset.

Definition 5: A summary of CAR filter be made from rule thing $R$ where

$\operatorname{conf}(\mathrm{R})=(\operatorname{suppcount}(\mathrm{R}) / \operatorname{actoccr}(\mathrm{R}))_{-}$minconf.

Definition 6: If a social occasion of rules all have a comparable standard trailblazer, a classifier is made by mentioning the rules according to the organization underneath.

A standard R1has need over a standard R2if and just if:

(1)ifconf(R1) >conf(R2).

(2) $\operatorname{ifconf}(\mathrm{R} 1)=\operatorname{conf}(\mathrm{R} 2)$ and $\operatorname{supp}(\mathrm{R} 1)>\operatorname{supp}(\mathrm{R} 2)$.

(3) ifconf $(\mathrm{R} 1)=\operatorname{conf}(\mathrm{R} 2)$ and $\operatorname{supp}(\mathrm{R} 1)=\operatorname{supp}(\mathrm{R} 2)$ and

$\mathrm{R} 1$ is made before R2.

\section{Analysis Method}

CBA might be utilized to learn action design relationship, as we recommended. Patients' conduct accounts will be utilized as information. The exactness rules, just as help and certainty techniques, were created. The periods of the examination are portrayed in Figure 1.1.

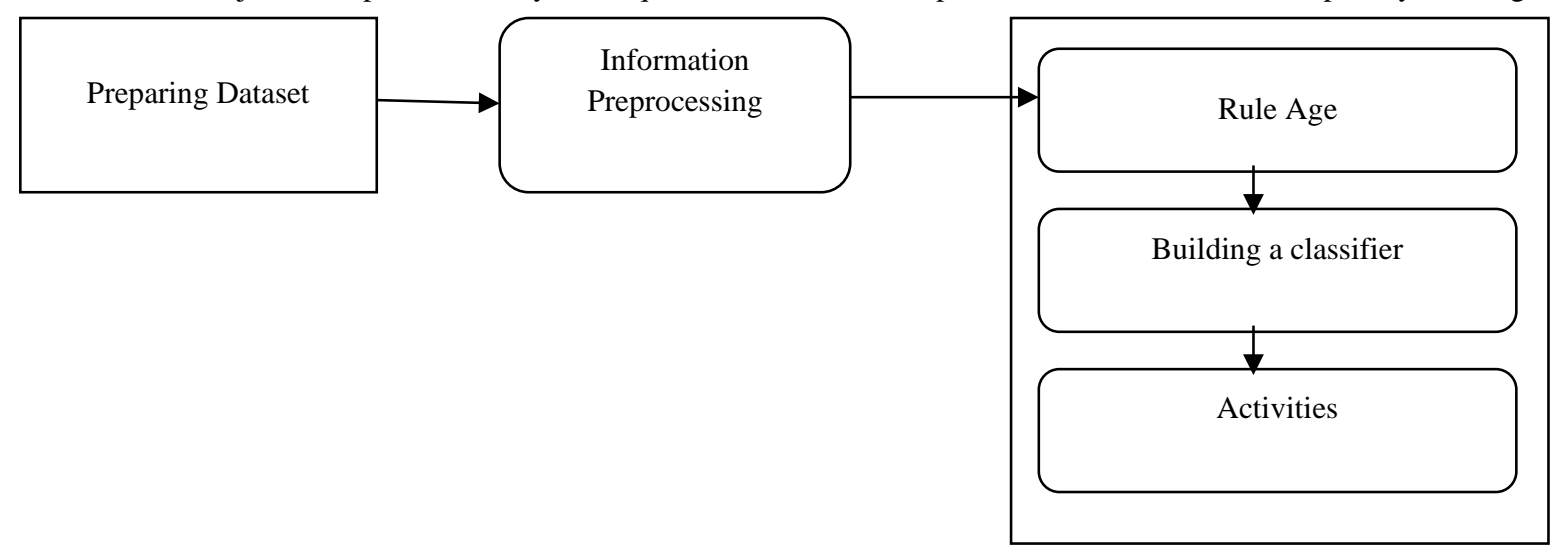

Figure 1. Examination Methodology Overview

The fundamental gathering and observational interpretations of exercises are numerically changed. Various kinds of information require distinctive standardization and significance planning strategies. Subsequent to preprocessing the information, we utilized CBA similarly as expressed in areas $2 \mathrm{C}$ and $3 \mathrm{C}$.Brute power is utilized to evaluate the best help and limit esteems. These numbers are picked to improve the classifier's precision. A k-crease cross approval technique was utilized to prepare the classifier model. After the classifier has been created, it is scrutinized. The preparation dataset is likewise used to make the model, with the precision assessed utilizing information from the testing assortment.

By utilizing the K-crease technique, you get high exactness with low predisposition and difference. $\mathrm{k}$ is set to 3 in our test. D1, D2, and D3 are three fundamentally unrelated subsets of the datasets that are haphazardly separated. The whole of the sets are about a comparative size. The arrangement and testing measure is repeated on various occasions, with one subset filling in as a test sample.D1 is used for preparing and D2 and D3 are used for planning in the first round. In resulting rounds, D2 and D3 are used to evaluate.

Minconf of $100 \%$ and minsupp of $30 \%$ were gotten from our mental imbalance dataset. With these figures, the most elevated exactness of $95.65 \%$ was accomplished altogether three rounds, with a normal precision of 85.27 percent. At last, there are seven principles for building a programmed classifier. In any case, for the default class, which is known as PDD-NOS, the entire plan of rules is named mental lopsidedness. Table II shows the principles in real life.

Mentally unbalanced youngsters' manifestations are extremely different. Our examination intends to distinguish themost widely recognized social patterns in Thailand. We can research the connection between these practices by separating the made principlesinto standard models as characterized in the DSM-IV. The laws of the relationship are portrayed in Figure 2. 
The two forerunners in rules \#3, \#4, and \#5 are from a similar DSM-IV sub-class: inability to build up peer connections adequate to formative stage and postponement in, or complete absence of, improvement of communicated in language, as demonstrated in the Figure.

\section{Created from CBA}

\begin{tabular}{|c|c|c|c|}
\hline Rules Antecedent & Rules class & Support (\%) & Confidence (\%) \\
\hline 7,12 & Mental Depression & 45 & 100 \\
\hline 3,12 & Mental Depression & 42 & 100 \\
\hline 1,16 & Mental Depression & 37 & 100 \\
\hline 2,16 & Mental Depression & 35 & 100 \\
\hline 1,20 & Mental Depression & 35 & 100 \\
\hline 19 & Mental Depression & 34 & 100 \\
\hline 22 & Mental Depression & 29 & 100 \\
\hline
\end{tabular}

\section{Table II: Classifier Rules}

\section{PRELIMINARY RESULT AND INTERPRETATION}

It is basic to unwind backing and trust to survey the 7 made guidelines. Since the trust for all guidelines in Table II is $100 \%$, a mix of results in each standard basically exists in a mental lopsidedness class, not PDD-NOS. Taking everything into account, in our dataset, these obvious direct models are essentially seen in remedially free users.As found in the models under.

rules can be deciphered as follows:

Rule \#1: $\{712\}$ Autism [supp $=45 \%$, conf $=100 \%$ ]

This standard proposes that for $45 \%$ of cases in the dataset:

IF lack of unconstrained utilization of clear movements toward pass on Also, inability to react to the grinning of others THEN set apart as compound imbalance

In the DSM-IV, the impedance direct "shortage of unconstrained utilization of major headways to pass on" takes a gander at to a sub-class called "nonappearance of utilizing changed nonverbal practices." This insufficiency regularly co-happens with the DSM-IV shortcoming, "weakness to respond to others' grinning" or "nonattendance of social or enthusiastic correspondence" in our dataset.

IV. This model didn't show in any PDD-NOS clients in our dataset.

Rule \#2: $\{312\}$ Autism [supp $=42 \%$, conf $=100 \%$ ]

This standard proposes that for $42 \%$ of cases in the dataset:

IF impairment in the capacity to start aconversation with others

Moreover, weakness to react to the grinning of others

By then set apart as compound imbalance

A huge part of mentally unbalanced individuals experience gives passing on and pulling in with others. Weakness to react to others' grins is routinely related with a deficit of status to begin a discussion with others.

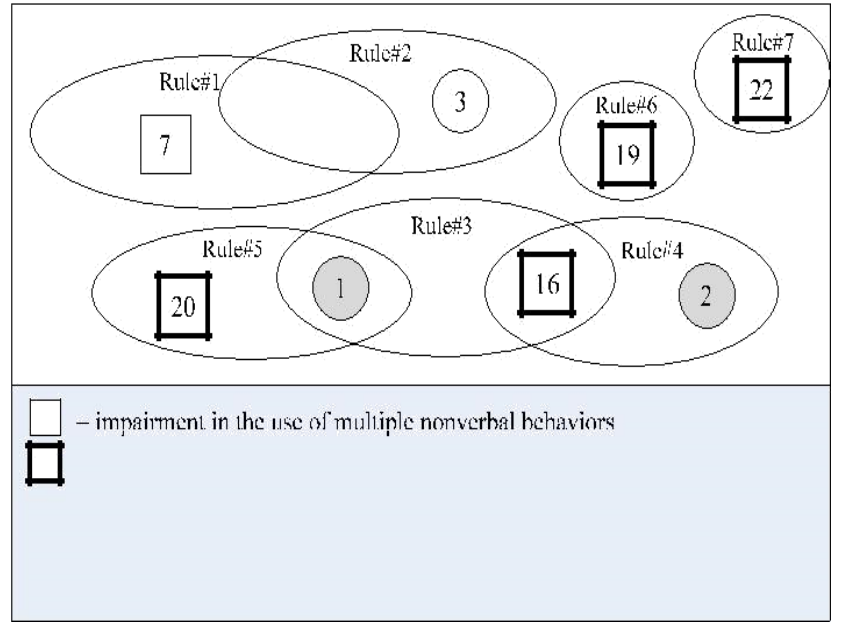




\section{Figure 2. Rules Descriptions}

There is no fascinating connection between lack practices with regards to PDD-NOS, as indicated by the discoveries. This is because of the way that our dataset has the least PDD-NOS patient data. Besides, in our dataset, all connections that happen in PDD-NOS records additionally exist in medically introverted records, making it hard to determine a PDD-NOS rule set.Nonetheless, the discoveries lead to some charming decisions about the qualifications among chemical imbalance and PDD-NOS, as itemized in this part.

Practice with mentally unbalanced and PDD-NOS kids has confirmed our discoveries. It has been concluded that CBA will be utilized to make a specialist structure that will remember urgent references for current clinical practices.

\section{END AND FUTURE STUDY}

MENTAL DEPRESSION patient data records can be researched using our structure. The resulting classifier has a critical level of precision in perceiving mental irregularity and PDD-NOS individual lead guidelines. Besides, the association among restoratively independent and PDD-NOS customers' guidelines of direct can be recognized. A condition construction can be expected with a genuine degree of sureness given a collection of insufficiencies. Regardless, there two or three deformities in the assessment: - Lack of clinical confirmation from standard customers for use in the planning stage $\bullet$ Prediction botch in conditions where a set number of tests appears to overfit the course of action At last, the use of familiar game plan in clinical applications changesenormously. Certain pieces of our investigation method are used in finding as additional arrangement records are amassed. In the near future, a MENTAL DEPRESSION data base and expert system can be developed.145-162 in Studies in Intellectual Disabilities 28, 2007.

\section{REFERENCES}

1. The American Psychiatric Association, “Autistic disorder”, Diagnostic and Statistical Manual of Mental Disorders, Fourth Edition Text Revision (DSM-IV-TR), 2000.

2. Murugesan, M., Thilagamani, S. ," Efficient anomaly detection in surveillance videos based on multi layer perception recurrent neural network", Journal of Microprocessors and Microsystems, Volume 79, Issue November 2020, https://doi.org/10.1016/j.micpro.2020.103303

3. C. Lord, M. Rutter, and S. Goode, "Autism Diagnostic Observation Schedule: a standardized observation of communicative and social behavior (ADOS)”, Journal of Autism and Developmental Disorders 19 (2), 1989, pp. 185-212.

4. Thilagamani, S., Nandhakumar, C. ." Implementing green revolution for organic plant forming using KNN-classification technique", International Journal of Advanced Science and Technology, Volume 29, Isuue 7S, pp. 1707-1712

I. Noens, I. van Berckelaer-Onnes, R. Verpoorten, and G. van Duijn, "The ComFor: An instrument for the indication of augmentative communication in people with autism and intellectual disability”, Journal of Intellectual Disability Research 50, 2006, pp. 621-632.

5. Thilagamani, S., Shanti, N., " Gaussian and gabor filter approach for object segmentation”, Journal of Computing and Information Science in Engineering, 2014, 14(2), 021006, https://doi.org/10.1115/1.4026458.

6. Y. Qiang, Y. Guo b X. Li, Q. Wanga, H. Chenc, and D. Cuic, "The diagnostic rules of peripheral lung cancer preliminary study based on data mining technique”, Journal of Nanjing Medical University, 2007, 21(3), pp. 190-195.

7. S. Palaniappan and R. Awang, "Intelligent heart disease prediction system using data mining techniques", IJCSNS International Journal of Computer Science and Network Security, VOL.8 No.8, August 2008.

8. B. Liu, W. Hsu, and Y. Ma, "Integrating classification and association rule mining”, Knowledge Discovery and Data Mining, NY, AAAIPress, 1998, pp. 80-86.

9. Rhagini, A., Thilagamani, S. , "Women defence system for detecting interpersonal crimes",International Journal of Advanced Science and Technology, 2020, Volume 29,Issue7S, pp. 1669-1675

10. X. Yin, and J. Han, “CPAR: Classification based on predictive association rule”, Proceeding of the SIAM International Conference on Data Mining, San Francisco, CA: SIAM Press, 2003, pp. 369-376.

11. F. Thabtah, "Review on associative classification mining”, Journal of Knowledge Engineering Review, Cambridge Press, Vol.22:1, 2007, pp. 37-65.

12. K.Deepa, S.Thilagamani, "Segmentation Techniques for Overlapped Latent Fingerprint Matching”, International Journal of Innovative Technology and Exploring Engineering (IJITEE), ISSN: 2278-3075, Volume-8 Issue-12, October 2019. DOI: 10.35940/ijitee.L2863.1081219.

13. Coenen, F., 2004, “LUCS KDD implementation of CBA (Classification Based on Associations)”, LUCS KDD, Department of Computer Science, The University of Liverpool, $U K$.

14. Deepa. $K$, LekhaSree. $R$, Renuga Devi. B, Sadhana. V, Virgin Jenifer. S, “Cervical Cancer Classification”, International Journal of Emerging Trends in Engineering Research, 2020, 8(3), pp. 804-807 https://doi.org/10.30534/ijeter/2020/32832020

15. Y. Liu, S. Zhao, Q. Wang, and Q. Gao, “Learning more distinctive representation by enhanced PCA network, ' Neurocomputing, vol. 275, pp. 924-931, Jan. 2018.

16. L. He and C. Cao, “Automated depression analysis using convolutional neural networks from speech,'”J. Biomed. Informat., vol. 
83, pp. 103-111, Jul. 2018.

17. Santhi,P., Mahalakshmi, G.,Classification of magnetic resonance images using eight directions gray level co-occurrence matrix (8dglcm)based feature extraction, International Journal of Engineering and Advanced Technology,2019,8(4), pp.839-846.

18. W. Sun, S. Xie, J. Yu, L. Niu, and W. Sun, 'Classification of thyroid nodules in ultrasound images using deep model based transfer learning and hybrid features, ', in Proc. IEEE Int. Conf. Acoust., Speech Signal Process. (ICASSP), Mar. 2017, pp. 919-923.

19. Santhi, P., Priyanka, T.,Smart India agricultural information reterival system, International Journal of Advanced Science and Technology, 2020, 29(7 Special Issue), pp. 1169-1175.

20. L. Zheng, S. Wang, L. Tian, F. He, Z. Liu, and Q. Tian, “Query-adaptive late fusion for image search and person re-identification, '” in Proc. IEEE Conf. Comput. Vis. Pattern Recognit. (CVPR), Jun. 2015, pp. 1741-1750.

21. X. Zhang, H. Zhang, Y. Zhang, Y. Yang, M. Wang, H. Luan, J. Li, and T.-S. Chua, "Deep fusion of multiple semantic cues for complex event recognition, ' IEEE Trans. Image Process., vol. 25, no. 3, pp. 1033-1046, Mar. 2016.

22. Vijayakumar, P, Pandiaraja, P, Balamurugan, B \& Karuppiah, M 2019, 'A Novel Performance enhancing Task Scheduling Algorithm for Cloud based E-Health Environment', International Journal of E-Health and Medical Communications, Vol 10,Issue 2,pp 102-117.

23. Y. Qi, Y. Wang, X. Zheng, and Z. Wu, “Robust feature learning by stacked autoencoder with maximum correntropy criterion,'” in Proc. IEEE Int. Conf. Acoust., Speech Signal Process. (ICASSP), May 2014, pp. 6716-6720.

24. P. Pandiaraja, N Deepa 2019 ," A Novel Data Privacy-Preserving Protocol for Multi-data Users by using genetic algorithm", Journal of Soft Computing, Springer, Volume 23 ,Issue 18, Pages 8539-8553.

25. X. Yuan, B. Huang, Y. Wang, C. Yang, and W. Gui, "Deep learning-based feature representation and its application for soft sensor modeling with variable-wise weighted SAE, "' IEEE Trans. Ind. Informat., vol. 14, no. 7, pp. 3235-3243, Jul. 2018.

26. N Deepa , P. Pandiaraja, 2020 ," Hybrid Context Aware Recommendation System for E-Health Care by merkle hash tree from cloud using evolutionary algorithm”, Journal of Soft Computing, Springer, Volume 24 ,Issue 10, Pages 7149-7161.

27. C. Tao, H. Pan, Y. Li, and Z. Zou, "Unsupervised spectral-spatial feature learning with stacked sparse autoencoder for hyperspectral imagery classification, ' IEEE Geosci. Remote Sens. Lett., vol. 12, no. 12, pp. 2438-2442, Dec. 2015.

28. N Deepa, P. Pandiaraja, 2020, "E health care data privacy preserving efficient file retrieval from the cloud service provider using attribute based file encryption “, Journal of Ambient Intelligence and Humanized Computing , Springer , https://doi.org/10.1007/s12652-020-01911-5.

29. J. Dai, H. Song, G. Sheng, and X. Jiang, "Cleaning method for status monitoring data of power equipment based on stacked denoisingautoencoders, ' IEEE Access, vol. 5, pp. 22863-22870, Aug. 2017.

30. Y. Lei, W. Yuan, H. Wang, Y. Wenhu, and W. Bo, “A skin segmentation algorithm based on stacked autoencoders, ' IEEE Trans. Multimedia, vol. 19, no. 4, pp. 740-749, Apr. 2017.

31. Y. Qi, C. Shen, D. Wang, J. Shi, X. Jiang, and Z. Zhu, "Stacked sparse autoencoder-based deep network for fault diagnosis of rotating machinery, '” IEEE Access, vol. 5, pp. 15066-15079, Jul. 2017.

32. L. Wang, Z. Zhang, and J. Chen, "Short-term electricity price forecasting with stacked denoisingautoencoders, "' IEEE Trans. Power Syst., vol. 32, no. 4, pp. 2673-2681, Jul. 2017.

I. M. Van Vliet and E. De Beurs, "The MINI-international neuropsychiatric interview. A brief structured diagnostic psychiatric interview for DSMIV en ICD-10 psychiatric disorders, ”' TijdschriftVoorPsychiatrie, vol. 49, no. 6, pp. 393-397, Jul. 2007.

A. M. Vijay 'Diagnostic and statistical manual of mental disorders, ' Psychiatry Res., vol. 189, no. 1, pp. 158159, Jan. 2011.

B. Gorris, A. R. Maccarini, F. Vanoni, and M. Poggioli, “Acoustic analysis of normal voice patterns in Italian adults by using praat, 'J. Voice, vol. 34, no. 6, pp. 9-18, May 2019.

33. K Sumathi, P Pandiaraja 2019," Dynamic alternate buffer switching and congestion control in wireless multimedia sensor networks", Journal of Peer-to-Peer Networking and Applications, Springer, Volume 13,Issue 6,Pages 2001-2010.

34. H. Larochelle, Y. Bengio, J. Louradour, and P. Lamblin, “Exploring strategies for training deep neural networks, ’ J. Mach. Learn. Res., vol. 1, pp. 1-40, Jan. 2009.

35. G. E. Hinton, S. Osindero, and Y.-W.Teh, “A fast learning algorithm for deep belief nets,'” Neural Comput., vol. 18, no. 7, pp. 1527-1554, Jul. 2006.

36. L. Jianhai, L. U. Kai, and S. Yuxuan, “A novel relieffeature selection algorithm based on mean-variance model, ' J. Inf. Comput.al Sci., vol. 8, no. 16, pp. 3921-3929, Dec. 2011.

37. V. Mitra, E. Shriberg, D. Vergyri, B. Knoth, and R. M. Salomon, “Crosscorpus depression prediction from speech,' in Proc. IEEE Int. Conf. Acoust., Speech Signal Process. (ICASSP), Apr. 2015, pp. 4769-4773.

38. W. Pan, “Depression recognition based on speech,'” Sci. Bull., vol. 63, no. 20, pp. 2081-2092, Sep. 2018.

39. Shankar, A., Pandiaraja, P., Sumathi, K., Stephan, T., Sharma, P. ," Privacy preserving E-voting cloud system based on ID based 
encryption " Journal of Peer-to-Peer Networking and Applications, Springer, https://doi.org/10.1007/s12083-020-00977-4.

40. H. Kaya, F. Eyben, A. A. Salah, and B. Schuller, "CCA based feature selection with application to continuous depression recognition from acoustic speech features, ' in Proc. IEEE Int. Conf. Acoust., Speech Signal Process. (ICASSP), May 2014, pp. 49.

41. H. Jiang, B. Hu, Z. Liu, L. Yan, T. Wang, F. Liu, H. Kang, and X. Li, “'Investigation of different speech types and emotions for detecting depression using different classifiers, ' Speech Commun., vol. 90, pp. 39-46, Jun. 2017.

42. Y. Liu, S. Zhao, Q. Wang, and Q. Gao, 'Learning more distinctive representation by enhanced PCA network, ' Neurocomputing, vol. 275, pp. 924-931, Jan. 2018.

43. L. He and C. Cao, “Automated depression analysis using convolutional neural networks from speech," J. Biomed. Informat., vol. 83, pp. 103-111, Jul. 2018.

44. T. Majtner, S. Yildirim-Yayilgan, and J. Y. Hardeberg, "Combining deep learning and hand-crafted features for skin lesion classification, ', in Proc. 6th Int. Conf. Image Process. Theory, Tools Appl. (IPTA), Dec. 2016, pp. 1-6.

45. W. Sun, S. Xie, J. Yu, L. Niu, and W. Sun, "Classification of thyroid nodules in ultrasound images using deep model based transfer learning and hybrid features, ' in Proc. IEEE Int. Conf. Acoust., Speech Signal Process. (ICASSP), Mar. 2017, pp. $919-923$.

46. C. Wang, A. Elazab, J. Wu, and Q. Hu, 'Lung nodule classification using deep feature fusion in chest radiography, ' Computerized Med. Imag.Graph., vol. 57, pp. 10-18, Apr. 2017. 